\title{
MAGNETO-OPTIC STUDIES OF COMPOSITIONALLY MODULATED DyFe FILMS
}

\author{
R.Carey, L.Dieu, D.M.Newman and B.W.J.Thomas \\ Centre for Data Storage Materials, Coventry University, Coventry CV1 5FB
}

\begin{abstract}
Magneto-optic measurements of the anisotropy [5] of multilayer DyFe films of constant composition, as a function of Dy layer thickness, confirms a thaximum in perpendicular anisotropy for films containing monolayers of Dy. The results are discussed briefly in terms of available models for the origin of anisotropy in multilayers [6] and thin RE/TM films with non-S-state RE atoms [9].
\end{abstract}

\section{INTRODUCTION}

Amorphous heavy rare earth-transition metal(RE-TM) alloys are currently used as the active layer in magneto-optic data storage discs. The particular properties that make them so attractive have been well documented, see for example [1]. In the ever continuing search for improved, possibly less expensive and more versatile storage materials the role of multilayer RE-TM structures is becoming increasingly significant [2], as is the replacement of $\mathrm{Tb}$ by the less expensive Dy [3].

Perhaps the most significant feature of this alternative strategy for improved RE-TM storage materials is the greatly increased perpendicular anisotropy obtained by deliberately modulating the composition of these alloys in layered structures. A clearer understanding of the origin of this increased anisotropy is clearly desirable and is the main aim of the work reported here.

In a previous report [4] the magnetic properties of DyFeCo thin films were examined as a function of multilayer structure. In this report a magneto-optic technique [5], developed for the measurement of perpendicular anisotropy, is used to examine more closely the variation of anisotropy produced as a result of controlled composition modulation in simple binary DyFe amorphous layers. The significance of the model proposed in [6] to these RE-TM multilayer structures is discussed briefly.

\section{EXPERIMENTAL TECHNIQUES}

Amorphous DyFe films were prepared using the same technique as that described in detail in [4]. By varying the rotation rates over the range 4-16 rpm the set of samples detailed in Table 1 was prepared with composition $\left(\mathrm{Dy}_{18} \mathrm{Fe}_{82}\right.$ ) and total film thickness (70nm) kept constant throughout. Each sample is protected on both its substrate and free surface by a sputtered $15 \mathrm{~nm}$ silicon nitride film. In this Table, as well as in subsequent captions and labelling of axes, "rotation rate" refers to the rotation rate of the substrate table during deposition.

Concentration across the range of structured samples was monitored by energy dispersive Xray analysis and magnetic compensation temperature measurement. For these ferrimagnetic samples the latter is a sensitive indicator of composition variation. As the compensation temperature for the composition selected for this investigation is below room temperature, measurement of this critical temperature required the use of a cryogenic stage in the polar Kerr hysteresis loop plotter used to monitor the magnetic properties of the films. Saturation magnetisation measurements were made using a vibrating sample magnetometer.

The perpendicular anisotropy associated with each of the films was measured using the magneto-optic technique reported in [5]. This involves the measurement, at a suitable angle of incidence, of the transverse Kerr effect as a function of the in-plane, applied magnetic field and the subsequent interpolation of values for the perpendicular anisotropy constants $\mathrm{K}_{1}$ and $\mathrm{K}_{2}$ for each of the samples.

\section{RESULTS}

The variation of saturation magnetisation and coercivity as a function of substrate rotation rate are shown in Figure 1. The former is consistent with the anticipated constant composition whilst the latter is of the same form as that reported for DyFeCo in [4], showing a maximum coercivity developed in samples for 


$\begin{array}{lllllllll}\text { Rotation rpm } & 16 & 14 & 12 & 10 & 8 & 6 & 5 & 4 \\ \text { Dy layer (d)nm } & \mathbf{0 . 2 8} & \mathbf{0 . 3 2} & \mathbf{0 . 3 7} & \mathbf{0 . 4 5} & \mathbf{0 . 5 6} & \mathbf{0 . 7 5} & \mathbf{0 . 9 0} & \mathbf{1 . 1 2} \\ \text { Fe layer nm } & \mathbf{0 . 2 5} & \mathbf{0 . 2 9} & \mathbf{0 . 3 3} & \mathbf{0 . 4 0} & \mathbf{0 . 5 0} & \mathbf{0 . 6 7} & \mathbf{0 . 8 0} & \mathbf{1 . 0 0} \\ \text { Bi-layer nm } & \mathbf{0 . 5 3} & \mathbf{0 . 6 1} & \mathbf{0 . 7 1} & \mathbf{0 . 8 5} & \mathbf{1 . 0 6} & \mathbf{1 . 4 2} & \mathbf{1 . 7 0} & \mathbf{2 . 1 3}\end{array}$

Table 1.

\begin{tabular}{|c|c|c|c|c|c|c|c|c|}
\hline pm & & 14 & 12 & 10 & 8 & 6 & 5 & 4 \\
\hline$M^{k}$ & $\begin{array}{l}3.25 \\
173.5\end{array}$ & $\begin{array}{l}5.47 \\
142.6\end{array}$ & $\begin{array}{l}9.45 \\
183.3\end{array}$ & $\begin{array}{l}11.46 \\
175.7\end{array}$ & $\begin{array}{l}8.25 \\
203.7\end{array}$ & $\begin{array}{l}8.48 \\
148.7\end{array}$ & $\begin{array}{l}1.40 \\
132.4\end{array}$ & $\begin{array}{l}0.2 \\
165\end{array}$ \\
\hline$\times 10^{5}$ & 4. & 5.18 & 10.77 & 12.01 & 11.01 & 7.70 & 2.03 & 1.83 \\
\hline cc) $\times 10^{5}$ & 0.70 & 0.98 & 1.30 & 1.51 & 1.26 & 0.95 & 0.93 & 0.25 \\
\hline
\end{tabular}

Table 2.

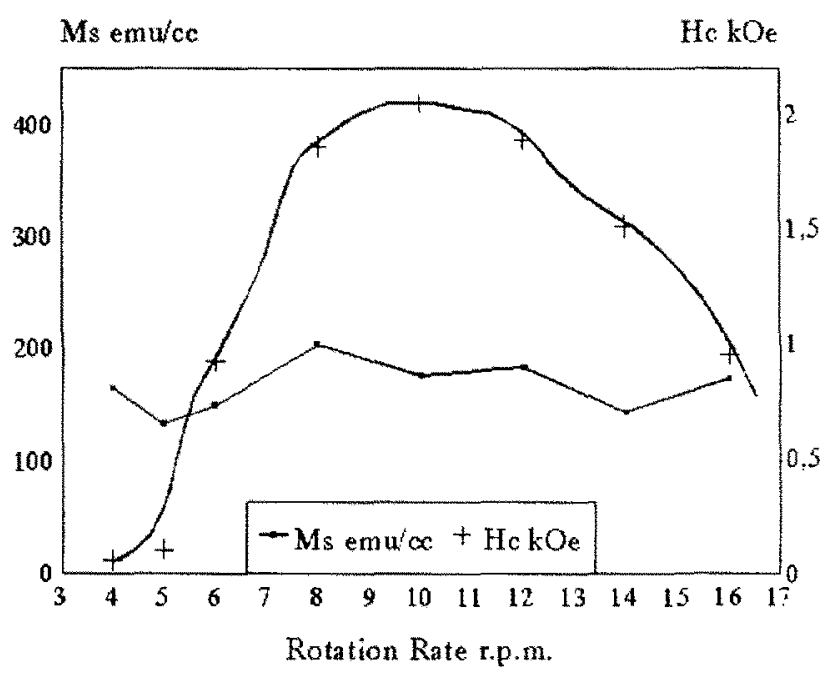

Figure 1: Saturation magnetization and coercivity as a function of substrate rotation rate

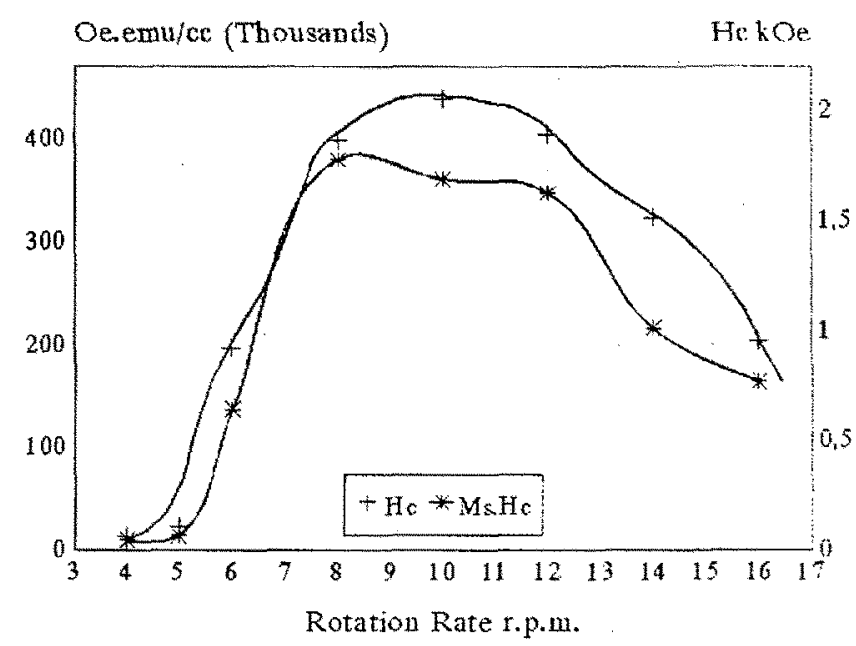

Figure 2: The $\mathrm{MH}_{\mathrm{c}}$ product and coercivity as a function of substrate rotation rate which monolayers of Dy are anticipated in the multilayer structure. The $\mathrm{M}_{\mathrm{s}} \mathrm{H}_{\mathrm{c}}$ product, shown together with the coercivity variation in Figure 2 , indicates the same maximum value for layered samples with monolayer thickness of Dy.

Data for Figures 1 and 2 are included in Table 2 , together with values obtained for $\mathrm{K}_{1}$ and $\mathrm{K}_{2}$ from the transverse Kerr effect measurements. $\mathrm{K}_{1}$ and $\mathrm{K}_{2}$ are shown as functions of substrate rotation rate in Figure 3 . Interestingly, although $\mathrm{K}$ is typically an order of magnitude greater than $\mathrm{K}$, for films with large perpendicular anisotropy, both exhibit broad maxima about the monolayer Dy structure. This result is perhaps clearer in Figure 4, where the total anisotropy energy density is plotted as a function of the Dy layer thickness.

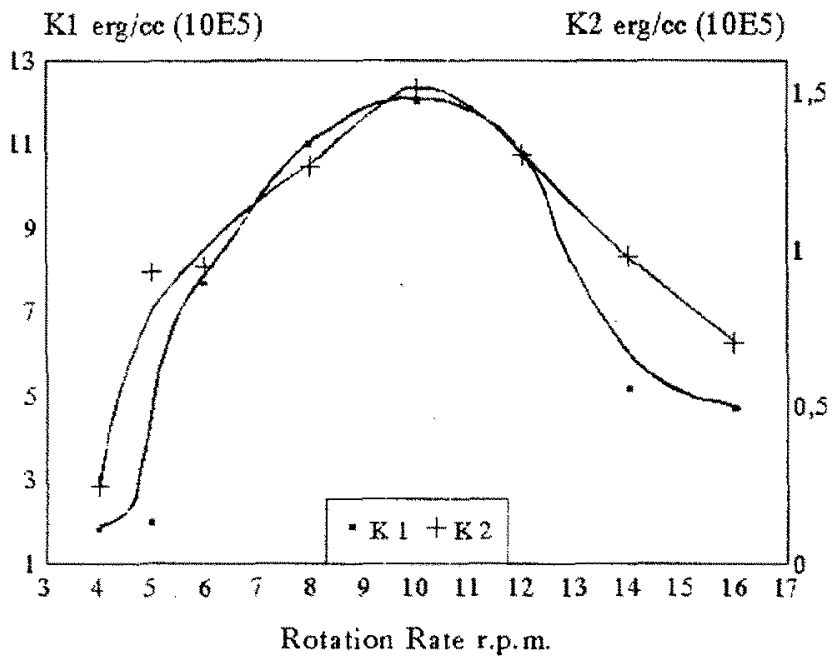

Figure 3: Anisotropy constants $K_{1}$ and $K_{2}$ as a function of substrate rotation rate 


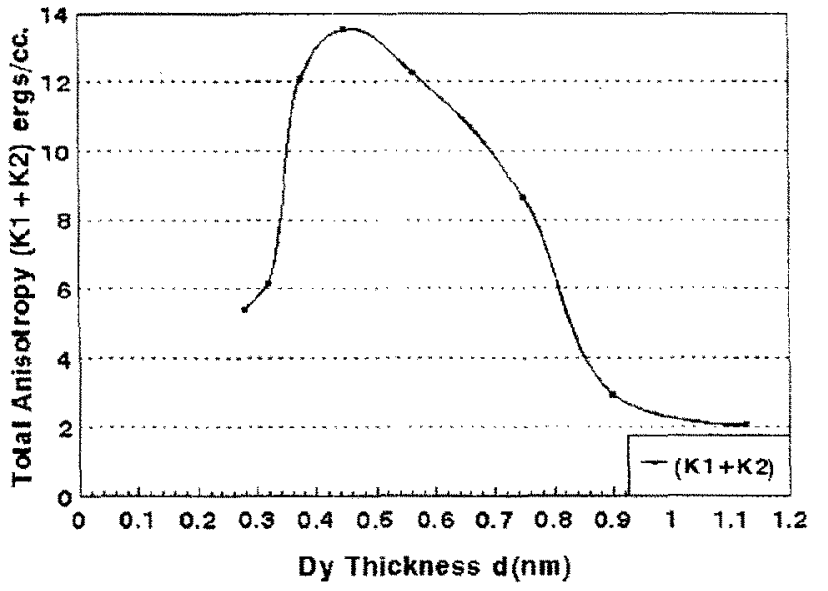

Figure 4: Total anisotropy energy density as a function of Dy layer thickness

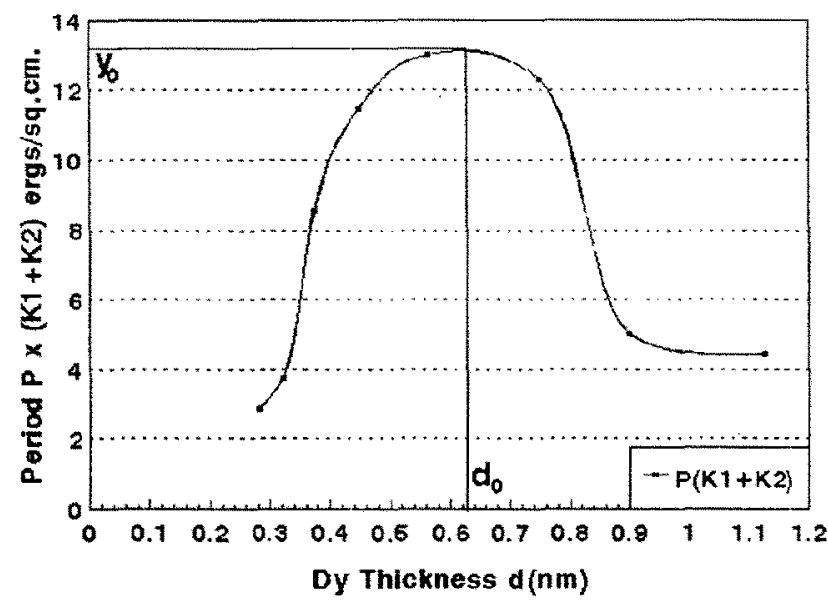

Figure 5: Product of total anisotropy energy density and bi-layer thickness as a function of Dy layer thickness

\section{DISCUSSION}

For the above investigation the film composition was chosen so that all samples could be saturated with the fields available within the laboratory. Thus a more complete picture of the behaviour of modulated films of DyFe could be obtained than was possible in [4].

The general features of the data presented above confirm the main tentative conclusion arrived at previously viz. the anisotropy of multilayer RE-TM films is a maximum for films in which the Dy is present in monolayers. This supports the general thesis put forward in
[2], that one would anticipate improved thermomagnetic storage capability for similarly optimised multilayer RE-TM films of the appropriate composition.

The magneto-optic determination of the anisotropy, [5], has proved to be very effective in this investigation, yielding interesting data for comparison with available models for anisotropy in multilayer films.

The model put forward by Carcia et al [6] for the origin of the perpendicular easy axis in $\mathrm{PdCo}$ multilayer films has been invoked by a number of authors (eg [7] and [8]) to produce, by extrapolation, anisotropy energies $\mathrm{K}_{\mathrm{i}}$ and $\mathrm{K}_{\mathrm{v}}$ for RE-TM multilayer films.

In the region where the anisotropy is a strong function of layer thickness the results presented in Figure 5 have the form:

$$
\mathrm{y}=\mathrm{P}\left(\mathrm{K}_{1}+\mathrm{K}_{2}\right)=\mathrm{y}_{0}-\mathrm{k}\left(\mathrm{d}-\mathrm{d}_{0}\right)^{2}
$$

as confirmed in Figure 6, where $\mathrm{P}$ is the bilayer period.

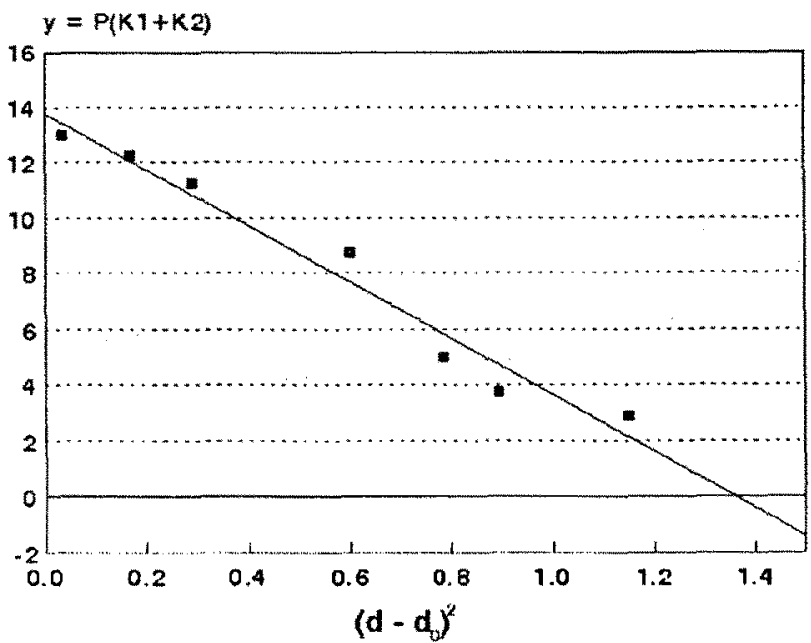

Figure 6: $P(K 1+K 2)$ fitted to equation 1

It is apparent that the model of Carcia et al [6] is not appropriate for DyFe films in the region of very thin bilayers (less than $2 \mathrm{~nm}$ ), where a maximum in total anisotropy energy is produced.

The increase in perpendicular anisotropy with changes in the layering structure is clearly related to the particular arrangement of nearest 
neighbours within the film and is further evidence of the significance of structural anisotropy in RE-TM alloy films in which TM and non$\mathrm{S}$-state RE atoms are combined [9]. This will be discussed further in a future report.

\section{REFERENCES}

[1] R.Carey,D.M.Newman and B.W.J.Thomas: J Phys D: Appl Phys,28,2207 (1995)

[2] S.Tanaka, T.Shimouma, A.Nakaoki, K.Aratani and M.Kaneko:J Magn Soc Jpn, 15, S1, 331 (1991)

[3] D.Raasch: IEEE Trans Mag, 29(1), 34 (1993)

[4] R.Carey, D.M.Newman, J.P.Snelling, B.W.J.Thomas and L. Dieu: JMMM, 148, 491 (1995)

[5] R.Carey, D.M.Newman and B.W.J.Thomas: J Phys D:Appl Phys,26,839 (1993)

[6] P.F.Carcia, A.D.Meinhaldt and A.Suna: Appl Phys lett, 47,(2), 178 (1985)

[7] Z.S.Shan, S.Nafis, K.D.Aylesworth and D.J.Sellmeyer: J Appl Phys,63,(8), 3218 (1988)

[8] S.Honda,S.Nishimura and T. Kusuda: IEEE Trans Mag, 23,(5), 4027 (1989)

[9] V.G.Harris, W.T.Elam and N.C.Koon: NATO ASI on High Density Recording, II Ciocco, Italy, (June, 1992) 